\title{
Long term mortality in critically ill burn survivors
}

\author{
SL Nitzschke ${ }^{1}$, AC Offodile II ${ }^{2}$ FK Gibbons ${ }^{3}$, A Salim ${ }^{1}$, KB Christopher $^{4^{*}}$ \\ From ESICM LIVES 2015 \\ Berlin, Germany. 3-7 October 2015
}

\section{Introduction}

Little is known about long term survival risk factors in critically ill burn patients who survive hospitalization.

\section{Objectives}

We hypothesized that patients with major burns who survive hospitalization would have favorable long term outcomes.

\section{Methods}

We performed a two center observational cohort study between 1998-2007 in 365 critically ill adult burn patients who survived to hospital discharge. The exposure of interest was major burn defined a priori as $>20 \%$ total body surface area burned [TBSA]. The modified Baux score was determined by age $+\%$ TBSA +17 (inhalational injury). The primary outcome was all-cause 5 year mortality based on the US Social Security Administration Death Master File. Adjusted associations were estimated through fitting of multivariable logistic regression models. Time-to-event analysis was performed using Cox proportional hazard regression.

\section{Results}

Of the cohort patients studied, $76 \%$ were male, $29 \%$ were non white, $14 \%$ were over $65,32 \%$ had TBSA $>20 \%$, and $45 \%$ had inhalational injury. The mean age was $45,92 \%$ had 2 nd degree burns, $60 \%$ had 3rd degree burns, 21\% received vasopressors, and $26 \%$ had sepsis. The mean TBSA was $20.1 \%$. The mean modified Baux score was 72.8 . Post hospital discharge 5 year mortality rate was $9.0 \%$. The 30 day hospital readmission rate was $4 \%$. Patients with major burns were significantly younger (41 vs 47 years) had a significantly higher modified Baux score (89 vs 62), and had significantly higher comorbidity, acute organ failure, inhalational injury and sepsis (all $\mathrm{P}<0.05)$. There were no differences in gender and the Acute Organ Failure score

\footnotetext{
${ }^{4}$ Brigham and Women's Hospital, Renal Division, Boston, United States
} Full list of author information is available at the end of the article between major and non-major burns. In a logistic regression model adjusted for inhalational injury, presence of $3^{\text {rd }}$ degree burn, gender and the Acute Organ Failure score, a validated ICU risk-prediction score derived from age, race, surgery vs. medical patient type, comorbidity, sepsis and acute organ failure covariates, major burn was associated with a 3 fold decreased odds of 5 year post-discharge mortality compared to patients with TBSA $<20 \%$ [OR $=0.29$ (95\%CI 0.11-0.78; $\mathrm{P}=0.014)]$. The adjusted model showed good discrimination [AUC 0.81 (95\%CI 0.74-0.89)] and calibration (Hosmer-Lemeshow $\chi^{2} \mathrm{P}=0.67$ ). Cox proportional hazard multivariable regression modeling, adjusting for inhalational injury, presence of $3^{\text {rd }}$ degree burn, gender and the Acute Organ Failure score, showed that major burn was predictive of mortality following hospital admission $[\mathrm{HR}=0.34$ (95\% CI 0.15-0.76; $\mathrm{P}=0.009)$ ]. The modified Baux score was not predictive for mortality following hospital discharge [OR 5 year post-discharge mortality = 1.00 (95\%CI 0.99-1.02;P = 0.74); HR for post-discharge mortality $=1.00(95 \% \mathrm{CI} 0.99-1.02 ; \mathrm{P}=0.55)]$.

\section{Conclusions}

Critically ill patients with major burns who survive to hospital discharge have decreased 5 year mortality compared to those with less severe burns. The modified Baux score was not predictive for long term outcomes following discharge.

\section{Authors' details}

'Brigham and Women's Hospital, Division of Trauma, Burn, and Surgical Critical Care, Boston, United States. ${ }^{2}$ Lahey Hospital and Medical Center, Department of Plastic and Reconstructive Surgery, Burlington, United States. ${ }^{3}$ Massachusetts General Hospital, Pulmonary and Critical Care Medicine, Boston, United States. ${ }^{\circledR}$ Brigham and Women's Hospital, Renal Division, Boston, United States.

Published: 1 October 2015

doi:10.1186/2197-425X-3-S1-A32

Cite this article as: Nitzschke et al: Long term mortality in critically ill burn survivors. Intensive Care Medicine Experimental 2015 3(Suppl 1):A32. (c) 2015 Nitzschke et al.; This is an Open Access article distributed under the terms of the Creative Commons Attribution License (http:// creativecommons.org/licenses/by/4.0), which permits unrestricted use, distribution, and reproduction in any medium, provided the original work is properly cited. 